\title{
Otimizando a perda de gordura corporal durante os exercícios
}

\author{
Antônio Felipe Correa Marangon ${ }^{1}$ \\ Alexis Fonseca Welker ${ }^{2}$
}

\begin{abstract}
RESUMO - Um dos principais objetivos da realização de exercícios físicos é a perda de gordura corporal. Antes de ocorrer a oxidação lipídica, a gordura é quebrada sob a ação de hormônios lipolíticos, liberando ácidos graxos e glicerol para o sangue. A queima de gordura depende de vários fatores, sendo que a oxidação relativa de ácidos graxos é maior em exercícios de baixa intensidade, mas a queima líquida de gordura alcança maiores valores em exercícios executados geralmente entre 55 e $72 \%$ do $\mathrm{VO}_{2 \max }$. A intensidade do exercício pode ser medida pelo consumo de oxigênio ou pela frequiência cardíaca.
\end{abstract}

Palavras-chave: metabolismo, gordura, nutrição, exercício.

\section{Optimizing body fat loss during exercises}

\begin{abstract}
One of the main purposes of realization of physical exercises is the body fat loss. Before the lipid oxidation, lipolytic hormones break the fat, resulting in fatty acids and glycerol in the blood. The lipid oxidation depends on various factors. The relative fatty acids oxidation is bigger during exercises of lower intensity, but the net fat oxidation reaches higher levels during exercises executed between 55 and $72 \%$ do $\mathrm{VO}_{2 \max }$. The exercise intensity can be measured by oxygen consumption or by the heart rate.
\end{abstract}

Key words: metabolism, lipid, nutrition, exercise.

1,2 Professores do curso de Fisioterapia da Faculdade de Ciências da Saúde, UniCEUB.

E-mail: felipemarangon@terra.com.br 
A prática de atividade física e a mudança de hábitos alimentares são duas maneiras bem conhecidas e usadas para alcançar objetivos específicos (Welker, 1999). Elas têm crescido nos últimos tempos, o que é percebido pelo aumento do número de academias de ginástica e de suplementos alimentares, divulgados na mídia. Tanto os exercícios físicos quanto a alimentação podem otimizar o aumento do desempenho físico ou a mudança da composição corporal (Kraemer \& Newton, 2000; Lambert \& Goedecke, 2003), sendo que para isto, é fundamental que eles sejam realizado com base em informações científicas (Clark, 1998). Vários são os objetivos que levam as pessoas a exercitarem-se como, por exemplo, aumento do desempenho, controle do estresse, melhoria da qualidade de vida e das condições cardiorespiratórias, mudança da composição corporal (estética) ou mesmo como forma de relaxamento.

A redução ponderal ou de gordura corporal são objetivos muito comuns, pois além da mudança estética, elas proporcionam vários benefícios, como redução da pressão arterial e aumento da captação de glicose pela maior sensibilidade à insulina (Welker \& Souza, 2002a; Duncan e cols., 2003), fazendo com que a prática de exercícios auxilie o controle glicêmico de pacientes portadores de Diabetes mellitus (Morais, 2003).

A perda de gordura pode ser otimizada durante exercícios executados em determinadas intensidades. Quanto maior é a intensidade de um exercício, maior é o gasto de energia obtido da oxidação de nutrientes e, conseqüentemente, maior é o consumo de oxigênio. Desta forma, a intensidade de um exercício é medida através do consumo de oxigênio (Carter e cols., 2002; Ducan e cols., 2003). Por exemplo, exercícios de baixa intensidade, como uma caminhada, proporcionam um menor consumo de oxigênio que uma corrida em alta velocidade. A determinação do consumo de oxigênio é realizada através de calorimetria indireta, onde o ar inspirado pelo indivíduo entra em contato com um analisador de gases que mede a quantidade de oxigênio que está efetivamente sendo consumida (Simonson \& Defronzo, 1990). O equipamento é de relativo alto custo e o protocolo experimental exige alguns cuidados, o que dificulta a sua utilização.

Já foi demonstrado haver relação entre consumo de oxigênio durante exercícios e a frequiência cardíaca (Bitar e cols., 1996; Treuth e cols., 1998; Novas e cols., 2003). Desta forma, a medição da frequiência cardíaca (que pode ser feita com um simples relógio de pulso) poderia ser um método mais barato para controlar a execução de exercícios em intensidades que otimizem a queima de gordura, apesar de que ainda há dúvidas sobre qual seria a freqüência cardíaca que estaria relacionada com uma maior perda de gordura subcutânea como fonte energética, visto que a literatura apresenta valores distintos (Hawley, 2002). Mesmo existindo essa possibilidade, há um número significativo de indivíduos que realizam atividade 
física com objetivo de redução ponderal sem o controle de sua respectiva frequiência cardíaca (Branco e cols., 2003), mesmo sendo um fator importante para avaliar a fonte energética utilizada durante a prática desportiva (Novas e cols., 2003).

Quando o objetivo principal do praticante de atividade física é estético, seu intuito com a prática desportiva geralmente é a redução do percentual de gordura, ou seja, a redução da quantidade de gordura corporal sub cutânea. Para isso, várias modalidades são indicadas como, por exemplo, natação, ciclismo, caminhadas, corridas, sendo que a corrida parece ser mais eficiente que o ciclismo na queima de gordura (Achten e cols., 2003). Alguns autores ainda sugerem a musculação como alternativa de redução do percentual de gordura, um objetivo que pode ser alcançado a longo prazo (Hunter e cols., 2000).

Vários estudos têm demonstrado a redução no percentual de gordura de acordo com o tipo de exercício utilizado. Porém, a utilização da gordura como fonte energética irá depender diretamente da intensidade e duração do exercício (Sánchez, 1994).

Dessa forma, ao consultar a literatura científica, percebemos que alguns autores indicam diferentes intensidades e durações de treinamento para a maior queima de gordura, não havendo um consenso na recomendação de freqüência cardíaca (FC) ideal para a oxidação de gordura como fonte energética. A maioria dos autores sugere uma faixa de treinamento baseada no consumo máximo de oxigênio $\left(\mathrm{VO}_{2 \max }\right)$. Todas estas informações podem proporcionar dúvidas entre aqueles que desejam realizar uma atividade física com objetivo de redução do percentual de gordura. Além disso, algumas pessoas não possuem acesso ao seu respectivo $\mathrm{VO}_{2 \max }$, não podendo utilizá-lo como base para seu treinamento. Aparentemente, a freqüência cardíaca talvez seja o método de mais fácil utilização durante os treinamentos como fator de correlação entre a intensidade do exercício e o metabolismo lipídico. Considerando que ainda não existe um consenso a respeito da intensidade de exercício que otimize a metabolização de gordura como fonte de energia (Sanchez, 1994; Bryner, 1997), uma avaliação dos dados existentes torna-se necessária.

\section{Revisão bibliográfica}

\section{Fontes energéticas}

A Tabela 1 demonstra as fontes energéticas presentes no organismo humano, além de suas respectivas quantidades calóricas. 
Tabela 1. Fontes de depósitos de substratos em seres humanos.

\begin{tabular}{lll}
\hline $\begin{array}{l}\text { COMBUSTÍVEL } \\
\text { Combustíveis Circulantes } \\
\text { Glicose }\end{array}$ & PESO & ENERGIA (Kj) \\
$\quad \begin{array}{l}\text { Ácidos Graxos Livres } \\
\text { (plasma) }\end{array}$ & 0,02 & 336 \\
$\quad 0,0004$ & 17 \\
$\quad \begin{array}{l}\text { Triglicerídios (plasma) } \\
\text { Depósitos Teciduais } \\
\text { Gordura }\end{array}$ & 0,004 & 168 \\
$\quad$ Tecido Adiposo & 15 & \\
$\quad$ Intramuscular & 0,3 & 588.000 \\
$\quad$ Proteínas & 10 & 11.760 \\
Glicogênio & 0,085 & 172.000 \\
$\quad$ Fígado & 0,35 & 1.470 \\
$\quad$ Músculo & & 6.090 \\
TOTAL & & 779.320 \\
\hline
\end{tabular}

Fonte: Robergs (2002).

As gorduras representam a maior reserva de energia do organismo, sendo encontrada na forma de triacilgliceróis no tecido adiposo e no plasma e na forma de ácidos graxos livres no sangue. O tecido adiposo constitui a forma de armazenamento de todo o excesso de nutrientes, quer este excesso seja oriundo dos carboidratos, proteínas ou mesmo dos próprios lipídios (Nelson \& Cox, 2002).

Cada triacilglicerol é formado por uma molécula de glicerol e três de ácidos graxos ligados entre si por ligações éster, daí também serem chamados de ácidos graxos esterificados. Os ácidos graxos necessários ao corpo humano para a obtenção de energia e construção das partes hidrofóbicas de várias biomoléculas, são obtidos pela dieta e pela própria biossíntese. Quantidades excessivas de proteínas e carboidratos da dieta são rapidamente convertidas a ácidos graxos e armazenados como triacilgliceróis (Mcgarry, 1998). Os triacilgliceróis formados e os vindos das gorduras dietéticas são armazenados nas células adiposas, ocupando a maior parte do seu volume celular (Guyton \& Hall, 2002; Nelson \& Cox, 2002).

\section{Hormônios e exercício físico}

Assim que o exercício físico é iniciado, sinais são enviados através das 
vias eferentes pelo sistema nervoso até os músculos em movimento, provocando adaptação metabólica ao exercício (Guyton \& Hall, 2002). Para que o músculo obtenha energia através da quebra da molécula de ATP, esta tem que ser produzida a partir da degradação de carboidratos, lipídios e proteínas (Nelson \& Cox, 2002). Isto é intensificado por alguns hormônios durante o exercício, como as catecolaminas (epinefrina e norepinefrina), glucagon, hormônio do crescimento e cortisol (Pastor e cols., 1999). A epinefrina, assim como o glucagon, liga-se a receptores beta-adrenérgicos que promovem a ativação da enzima lípase hormôniosensível, que promove a degradação lipídica (lipólise) no tecido adiposo (Nelson \& Cox, 2002). A quebra das moléculas de triacilgliceróis (gordura) encontradas no tecido adiposo libera ácidos graxos e glicerol na circulação, de forma que como a lipólise é aumentada durante o exercício, os níveis de ácidos graxos e glicerol no sangue ficam aumentados (Welker \& Souza, 2002c).

O hormônio do crescimento reduz a captação de glicose pelo tecido adiposo, disponibilizando-a o tecido muscular. Durante os exercícios, a insulina, que é o hormônio que inibe a lipólise, é menos produzido, isto é, não impede uma aumento da quebra das gorduras. A insulina é fundamental para a captação de glicose pelas células em situações normais, porém durante exercícios físicos, tal captação tornase relativamente independente de insulina, o que é percebido pela redução da glicemia (Welker \& Souza, 2002a; Morais, 2003). Esse efeito é explicado por uma translocação dos transportadores de glicose (GLUT 4) causada pelo exercício (Pastor e cols., 1999; Robergs, 2002).

\section{Metabolismo lipídico}

A principal função dos lipídios é fornecer energia para o organismo. Em primeiro lugar eles devem ser degradados em seus componentes básicos, ácidos graxos e glicerol, através da lipólise. O glicerol não é significativamente utilizado pela maioria dos tecidos, pois seu aproveitamento necessita da enzima glicerol quinase. O fígado apresenta tal enzima, onde o glicerol pode oxidado para a obtenção de energia ou ser convertido em glicose (Nelson \& Cox, 2002).

Os ácidos graxos são captados por diversos tecidos, especialmente os músculos durante o exercício, onde ele é convertido em acil CoA graxo (Maughan, 2000). Tais moléculas necessitam atravessar a membrana mitocondrial interna e chegar à matriz da mitocôncria, onde são oxidadas numa via denominada b-oxidação ou ciclo de Lynen. Porém, como elas são geralmente impermeáveis à membrana, o transporte é realizado pela enzima carnitina-acil transferase I (CAT-I) que torna sua entrada na matriz mitocondrial possível e conseqüente oxidação para a produção de ATP (Devlin, 1998). Através de uma série de reações b-oxidação, 
cada ácido graxo é degradado em moléculas de acetil-CoA, que podem ser oxidadas no ciclo de Krebs para gerar energia ou ser convertidas em corpos cetônicos (Nelson $\&$ Cox, 2002).

A degradação de carboidratos também geram acetil-CoA, que em excesso promove a formação de malonil-CoA, um precursor da síntese lipídica. Este inibe a carnitina acil transferase I, isto é, a entrada de ácidos graxos para a matriz mitocondrial e consequiente oxidação. Por isto, a ingestão de carboidratos pode inibir a queima de gordura (Welker \& Souza, 2002b). Assim, o excesso de acetilCoA devido a uma ingestão aumentada de nutrientes não somente promove uma inibição da oxidação lipídica como um aumento dos depósitos de triacilgliceróis (Maughan, 2000).

A hidrólise do triacilglicerol irá fornecer moléculas de ácidos graxos, que serão convertidos em energia, e glicerol, que por sua vez poderá ser captado pelo fígado e servir como precursor de glicose através da gliconeogênse (Devlin, 1998). Porém, a velocidade com que o fígado capta glicerol para a gliconeogênese é reduzida, fazendo com que sua concentração sérica esteja aumentada durante o processo de lipólise (Nelson \& Cox, 2002; Robergs, 2002). Além disso, como dito anteriormente, o glicerol não é aproveitado pela maioria dos tecidos. Portanto, a concentração plasmática de glicerol torna-se uma forma viável de avaliar a intensidade da lipólise durante o exercício, pois sua presença está diretamente relacionada com a lipólise (Yannis, 1999; Welker \& Souza, 2002c).

A concentração de ácidos graxos circulantes no plasma durante a atividade física é primeiramente dependente da lipólise, mas também da sua reesterificação em triacilgliceróis no tecido adiposo (Maughan, 2000), sendo uma boa medida da taxa líquida de lipólise, isto é, da quantidade de lipídios que estará sendo queimada (Welker \& Souza, 2002b). Em contraste, o glicerol vindo dos capilares do tecido adiposo depende principalmente da lipólise, sendo, consequientemente, um indicador preciso da taxa de lipólise quando comparado à concentração de ácidos graxos (Yannis, 1999; Robergs, 2002).

\section{Diferenças no metabolismo lipídico entre homens e mulhe- res}

Existem evidências de que a utilização de lipídios como fonte de energia durante o exercício de endurance pelas mulheres seja superior do que a utilização pelos homens (Romijn, 2000), pois alguns estudos demonstraram que elas possuem maior facilidade de utilizar os ácidos graxos como fonte de energia durante o exercício devido sua maior quantidade no organismo (Forburg, 1984; Tarnopolsky, 
1995). Comparadas com os homens, as mulheres tem demonstrado preferência no metabolismo lipídico durante exercícios de intensidade moderada (Tarnopolsky, 1990).

Entretanto, este fato não deve ser considerado válido para todas as situações, pois atletas masculinos com alto nível de treinamento, mesmo com baixos percentuais de tecido adiposo, possuem elevada metabolização de ácidos graxos durante o exercício físico (Romijn, 2000). Além disso, a utilização de lipídios como substratos energéticos aumenta à medida que se tem mais tempo de treinamento, atuando também como fonte energética durante a recuperação (Romijn, 1993).

\section{Metabolismo lipídico e $\mathrm{VO}_{2}$}

Os lipídios são considerados o principal substrato em exercícios físicos de baixa intensidade e longa duração. Vários pesquisadores concordam que a utilização relativa dos ácidos graxos como fonte de energia para a prática esportiva reduz com o aumento da intensidade do exercício, ao passo que sua utilização aumenta com a duração da mesma (Friedlander e cols., 1999; Robergs, 2002).

O substrato energético a ser utilizado durante a prática de exercícios físicos, como a oxidação de lipídios, depende da interação entre vários fatores, como a intensidade do exercício, sexo, o nível de treinamento, concentração de ácidos graxos livres plasmáticos, quantidade de carboidrato disponível e fatores hormonais (Wolinsky, 1996; Astorino, 2000; Hawley, 2002), de forma que nem todo exercício contará com os lipídios como principal fonte energética (Robergs, 2002).

A dieta tem papel fundamental na utilização dos nutrientes durante o exercício, pois a energia necessária para o movimento provém dos alimentos. Já a intensidade também contribui para determinação da fonte energética, visto que exercícios físicos em alta intensidade necessitam de suprimento rápido de energia, o que é conseguido através dos carboidratos. Por outro lado, exercícios com baixa intensidade, possuem como principal nutriente os lipídios (Maughan, 2000).

Durante exercícios de baixa a moderada intensidade, a maior parte das gorduras utilizadas como fonte de energia deriva da circulação, na forma de ácidos graxos livres. À medida que a intensidade da atividade aumenta, o metabolismo lipídico deixa de ser a principal fonte energética, ao passo que os carboidratos passam a representar maior contribuição (Astorino, 2000). Isso ocorre devido ao fato de a glicose ser mais rapidamente degradada que ácidos graxos e sua degradação ser independente da presença de oxigênio, isto é, quando o músculo está em hipóxia (Astorino, 2000; Maughan, 2000). 
Existe uma diferença entre a lipólise e a oxidação lipídica em exercícios de alta intensidade comparados aos exercícios de baixa intensidade. Mesmo que a intensidade do exercício aumente, promovendo maior gasto energético e maior taxa de lipólise, não podemos afirmar que a taxa de oxidação dos ácidos graxos liberados pela lipólise também será superior. Quanto maior for a intensidade de exercício, maior é a oxidação de glicose e menor é a dos ácidos graxos, que são então reesterificados no tecido adiposo (Astorino, 2000).

Em geral, a oxidação de lipídios é muito maior que a de carboidratos em exercícios de baixa intensidade, por exemplo, a 25 do $\mathrm{VO}_{2 \max }$ (Romijin et al., 1993). Porém, como o gasto de energia é pequeno nessa intensidade, a oxidação líquida de gordura é também pequena, sendo que a efetiva oxidação lipídica alcança valores máximos em exercícios executados entre 55 e $72 \%$ do $\mathrm{VO}_{2 \max }$ (Achten et al., 2002), apesar de que a oxidação de glicose também esteja elevada.

Tabela 2. Percentuais de $V O_{2 \max }$ nos quais ocorre maior metabolização lipídica segundo diferentes autores.

\section{Autor}

Romijn et al. (2000)

Tarnopolsky et. al.. (1990)

FAO, WHO (2000)

Astorino (2000)

Romijin et al. (1993)

Galbo (1983)

Wolinsky (1996)

\section{$\mathrm{VO}_{2}$}

$65 \%$

$65 \%$

$45 \%$

$75 \%$

$25-85 \%$

$60-65 \%$

$50 \%$

\section{Influência do treinamento no metabolismo lipídico}

Indivíduos treinados apresentam maiores quantidades de enzimas lipolíticas nas células musculares quando comparados com indivíduos sedentários. O treinamento, principalmente se for aeróbio, induz ao aumento da densidade de capilares no tecido adiposo, assim como sua capacidade de oxidação dos ácidos 
graxos. Dessa forma, indivíduos treinados iniciam o processo de oxidação lipídica previamente do que indivíduos sedentários, que devem permanecer mais tempo em atividade para metabolizar os lipídios (Friedlander, 1999).

Por fim, quanto maior for o nível de treinamento aeróbio do indivíduo (condicionamento físico), maior será a utilização de gordura como fonte de energia. Isto ocorre devido à aquisição celular de novas mitocôndrias e enzimas críticas do Ciclo de Krebs, consequiências do treino aeróbio (Wolinsky, 1996; McArdle, 1998; Robergs, 2002). Um aumento na quantidade de mitocôndrias e o incremento de seu volume proporcionam uma área de superfície maior para o recebimento de ácidos graxos e maior capacidade de utilizá-los no lugar da glicose (Gollnick, 1985).

\section{Ordem da utilização dos nutrientes}

Durante a atividade física todos os nutrientes são metabolizados, embora em proporções distintas, de acordo com a intensidade da atividade. Qualquer macronutriente, seja carboidrato, proteína ou lipídio, é capaz de gerar intermediários para serem oxidados no Ciclo de Krebs e gerar energia (Vander, 1981; Nelson \& Cox, 2002). Durante atividades físicas prolongadas, quando os estoques de glicogênio muscular e glicose sanguínea estão baixos, as proteínas são degradadas mais intensamente disponibilizando aminoácidos para o organismo. Os aminoácidos são degradados (desaminação) fornecendo cetoácidos que são convertidos em glicose ou oxidados no Ciclo de Krebs (Robergs, 2002). Quando os estoques de glicogênio hepáticos se tornam baixos e a glicemia tende a diminuir, o fígado passa a promover maior taxa de gliconeogênese, ou seja, a produção de glicose a partir de outras fontes que não o glicogênio, principalmente dos aminoácidos (Devlin, 1998). Estes podem até aumentar a glicemia quando em abundância (Alves, 2003). A gliconeogênese ocorre especialmente em exercícios com duração prolongada (Robergs, 2002), evitando quedas exageradas na glicemia e a sobrevivência mesmo após dias em jejum, sendo raros os animais que não são capazes de realizar tais processos (Freitas e cols., 2003).

Dessa forma, não podemos afirmar que carboidratos são metabolizados antes das gorduras e proteínas. Todos são utilizados como fonte energética ao mesmo tempo, variando apenas sua respectiva contribuição individual (Mcardle, 1998; Maughan, 2000). Em geral, parece que a utilização de combustível durante o exercício segue uma sequiência, na qual a oxidação acontece na seguinte ordem: glicogênio muscular, glicose sanguínea e ácidos graxos. Quando os estoques de carboidratos estão diminuídos, a glicemia tende a reduzir, pois os diferentes tecidos utilizam glicose constantemente para a geração de energia, sendo que alguns 
tecidos, como o cérebro, utilizam somente a glicose em situações normais (Guyton \& Hall, 2002). Para manter a glicemia relativamente estável, o organismo utiliza os aminoácidos provindos da proteólise, isto é, há um aumento da degradação de proteínas corporais quando a disponibilidade de glicose diminui, como no início do jejum (Nelson \& Cox, 2002).

Com a progressão da falta de carboidratos, o organismo passa a produzir significativamente mais corpos cetônicos a partir da degradação dos ácidos graxos provindos da lipólise e estes são utilizados por diferentes tecidos, como o cérebro, que então passam a consumir menos glicose. Desta forma, como há um relativo menor de glicose, o fígado passa a não produzir tanta glicose a partir de aminoácidos, ou seja, no jejum ou exercício prolongados, as proteínas são "poupadas" em detrimento das gorduras (Nelson \& Cox, 2002).

\section{Quociente respiratório e medição da perda de gordura}

$\mathrm{O}$ Quociente Respiratório $(\mathrm{QR})$ é relação entre o $\mathrm{CO}_{2}$ produzido e o $\mathrm{O}_{2}$ consumido e varia em resposta aos substratos utilizados como fonte de energia. Como o catabolismo dos carboidratos e lipídios difere no consumo de oxigênio e produção de dióxido de carbono, a razão entre o volume de síntese de dióxido de carbono e o consumo de oxigênio é utilizada para indicar a predominância do carboidrato ou gordura como substrato para o catabolismo energético. A oxidação de glicose gera maior produção de $\mathrm{CO}_{2}$ em relação a de lipídios (Robergs, 2002).

Como a oxidação de carboidratos é elevada quando a intensidade do exercício está alta, pelo fato de eles fornecerem energia em curto espaço de tempo, a produção de $\mathrm{CO}_{2}$ no exercício de alta intensidade é relativamente maior. Desta forma, exercícios físicos com intensidades elevadas possuem valores de $\mathrm{QR}$ altos, de aproximadamente 1,0. Por outro lado, em exercícios de baixa intensidade, o organismo utiliza prioritariamente os lipídios como fonte energética, sendo que sua oxidação gera pouco $\mathrm{CO}_{2}$ e exige um consumo relativamente alto de $\mathrm{O}_{2}$. Conseqüentemente, o QR apresenta valores próximos de 0,7 no exercício de baixa intensidade, ou mesmo no repouso, o que significa dizer que a produção de dióxido de carbono é menor que o consumo de oxigênio (Maughan, 2000; Robesgs, 2002).

$\mathrm{O}$ QR de 0,82 a 0,85 reflete fontes energéticas mistas, ou seja, tanto carboidratos como ácidos graxos são oxidados para obtenção de energia (Wolinsky, 1996). Para a medição da contribuição das proteínas como substrato energético (catabolismo protéico), pode-se realizar um simples cálculo, onde amostras de urina são coletadas, e a partir da quantidade de nitrogênio excretada, estima-se a perda de proteínas (Ferrannini, 1988). 
Tabela 3. participação das gorduras e carboidratos de acordo com o quociente respiratório.

\begin{tabular}{ccc}
\hline QR & \multicolumn{2}{c}{$\%$ de KCal Derivado de } \\
\hline 0,7 & Carboidratos & Gorduras \\
0,75 & 0 & 100 \\
0,8 & 15,6 & 84,4 \\
0,85 & 33,4 & 66,6 \\
0,9 & 50,7 & 49,3 \\
0,95 & 67,5 & 32,5 \\
1,0 & 84 & 16 \\
\hline
\end{tabular}

\section{Conclusões}

A otimização da perda de gordura corporal através dos exercícios depende de vários fatores, como o nível de treinamento, sexo, estado nutricional e da intensidade do exercício.

A lipólise aumenta com a intensidade dos exercícios devido à maior liberação de hormônios lipolíticos, aumentando a disponibilidade de ácidos graxos e glicerol no sangue. Ela parece ocorrer em maiores taxas em indivíduos mais treinados, em mulheres e quando há menor disponibilidade de carboidratos.

A oxidação lipídica também varia de acordo com esses fatores, sendo que ainda não há consenso a respeito da intensidade do exercício físico que potencialize a oxidação lipídica, apesar de os pesquisadores concordarem que a proporção da oxidação entre carboidratos e lipídios é alterada quando a intensidade do exercício é modificada. A oxidação relativa de ácidos graxos é maior em exercícios de baixa intensidade, mas a queima líquida de gordura alcança maiores valores em exercícios executados geralmente entre 55 e $72 \%$ do $\mathrm{VO}_{2 \max }$.

Por fim, em jejum ou exercícios prolongados, os corpos cetônicos (provindos da gordura) são utilizados no lugar da glicose, fazendo com que menos aminoácidos sejam utilizados na gliconeogênese e conseqüentemente, as proteínas sejam relativamente preservadas. 


\section{Referências}

ACHTEN, J.; GLEESON, M. \& JEUKENDRUP, A. E. Determination of the exercise intensity that elicits maximal fat oxidation. Med. Sci. Sports. Exerc., 34: 92-97, 2002.

ACHTEN, J.; VENABLES, M.C. \& JEUKENDRUP, A.E. Fat oxidation rates are higher during running compared with cycling over a wide range of intensities. Metabolism, 52:747-52, 2003.

ALVES, A.P.M. Resposta glicêmica à ingestão de solução de aminoácidos. Brasília: Faculdade JK, 2003. 29p. Monografia de Graduação em Nutrição.

ASTORINO, T. A. Is the ventilatory threshold coincident with maximal fat oxidation during submaximal exercise in women? J Sports Med Phys Fitness; 40: 209-16, 2000.

BITAR, A; VERMOREL, M.; FELLMANN, N.; BEDU, M.; CHAMOUX, A. \& COUDERT, J. Heart rate recording method validated by whole body indirect calorimetry in 10-yr-old children. J. Appl. Physiol., 81: 1169-1173, 1996.

BRANCO, F. C.; LIMA, J. R.; VIANNA, J. Freqüência cardíaca na prescrição de corredores de fundo. Revista Digital Vida \& Saúde. 2: 2003.

BRYNER, R. W.; TOFFLE, R. C.; ULLRICH, I. H. The effects of exercise intensity on body composition, wheight loss, ad dietry composition in women. J Am Coll Nutr, 16: 68-73, 1997

CARTER, H.; PRINGLE, J.S.; JONES, A.M. \& DOUST, J.H. Oxygen uptake kinetics during treadmill across exercise intensity domains. Eur. J. Appl. Physiol., 86: 347354, 2002.

DUNCAN, G. E.; PERRI, M. G.; THERIAQUE, D. W. Exercise training, without weight loss, increases insulin sensitivity and postheparin plasma lipase activity in previously sedentary adults. Diabetes Care, 26: 557-562, 2003.

FERRANNINI, E. The theoretical bases of indirect calorimetry: a review. Metabolism, 37: 287-301, 1998.

FREITAS, M. B.; PINHEIRO, E.; MILLAN, S. \& WELKER, A. Metabolic responses induced by fasting in common vampire bats Desmodus rotundus. J. Comp. Physiol. $B ., 2003$. in press.

FRIEDLANDER, A. L.; CASAZZA G. A.; HORNING, M. A. Endurance training increases fatty acid turnover but not fat oxidation, in young men. J. Appl. Physiol., 86: $2097-2105,1999$

FROBURG K.; PERSEN P. K. Sex differences in endurance capacity and metabolic response to prolonged, heavy exercise. J. Appl. Physiol., 52 : 446 - 450, 1984.

GALBO, H. Hormonal and metabolic adaptations to exercise. New York: Thieme Stratton Inc, 1983.

GOLLNICK, P. D. Metabolism of substrates: energy substrate metabolism during exercise 
and as modified by training. Fed. Proc., 44: 353-357, 1985.

GUYTON, A.C. \& HALL, J. Tratado de Fisiologia Médica. 10. ed. Rio de Janeiro: Guanabara Koogan, 2002.

HAWLEY, J. A Symposium: limits to fat oxidation by skeletal muscle during exercise. Med. Sci. Sports. Exerc., 34: 1475-1476. 2002.

HUNTER, G.R.; WETZSTEIN, C.J.; FIELDS, D.A.; BROWN, A. \& BAMMAN, M.M. Resistance training increases total energy expenditure and free-living physical activity in older adults. J. Appl. Physiol, 89:977-84, 2000.

KRAEMER, W.J. \& NEWTON, R.U. Training for muscular power. Phys. Med. Rehabil. Clin. N. Am., 11: 341-368, 2000.

LAMBERT, E.V. \& GOEDECKE, J.H. The role of dietary macronutrients in optimizing endurance performance. Curr. Sports. Med. Rep., 2: 194-201, 2003

MAUGHAN, R.; GLEESON, M. \& GREENHALF, P. L. Bioquímica do exercício de do treinamento.São Paulo: Manole, 2000.

MCARDLE, W. D., KATCH, F. I. \& KATCH, V. L. Fisiologia do exercício energia, nutrição e desempenho humano. 4 ed. Rio de Janeiro: Guanabara Koogan, 1998.

MORAIS, M. Avaliação do controle glicêmico através da colação e atividade física em uma criança portadora de Diabetes mellitus insulino-dependente. Brasília: Faculdade JK, 2003. 33p. Monografia de Graduação em Nutrição.

NELSON, D.L. \& COX, M.M. Lehninger princípios de bioquímica. 3. ed. São Paulo: Sarvier, 2002.

NOVAS, A.M.; ROWBOTTOM, D.G; \& JENKINS, D.G. A practical method of estimating energy expenditure during tennis play. J. Sci. Med. Sport., 6:40-50, 2003.

PASTOR, V. J.; RUIZ, M.; ACOSTA, A.M. \& AVILA, C. Metabolic and hormonal changes during aerobic exercise indistance runners. J. Physiol. Biochem, 55: 716, 1999.

ROBERGS, R. A. Princípios |Fundamentais de Fisiologia do Exercício: para aptidão, desempenho e saúde. São Paulo : Phorte, 2002

ROMIJN J. A.; COYLE E. F.; SIDOSSIS L. S.; ROSENBLATT J. \& WOLF R. R. Substrate metabolism during different exerecise intensities in endurance trained women. J. Appl Physiol., 88: 1707 - 1714, 2000

ROMIJN, J. A.; COYLE, E. F.; SIDOSSIS, L. S.; GASTALDELLI, A.; HOROWITZ, J. F. \& ENDERT E.; et al. Regulation of endogenous fat and carbohydrate metabolism in relation to exercise intensity and duration. Am J. Physiol., 265 : E380 -91, 1993.

ROMIJN, J.A; KLEIN, S. C. \& WOLF, R. Strenuous training increases lipolysis and triglyceride - fatty acid cycling at rest. J. Appl. Physiol., 75: 108 -113, 1993.

SANCHEZ, M. P.; GRUNEWALD, K. Body fat deposition: effects of dietary fat and two exercise protocols. Journal os the American Coolege os Nutrition, Vol. 13, ${ }^{\circ} 6$, 601-607, 1994.

SIMONSON, D. C. \& DEFRONZO, R. A. Indirect calorimetry: methodological and 
interpretative problems. Am. J. Physiol. Endocrinol. Metab., 258: E399 - 412, 1990.

TARNOPOLSKY, L. J.; MacDOUGALL J. D.; ATKINSON S. A. \& TARNOPOLSKY J. D. Carbohydrate loading and metabolism during exercise in men and women. $J$. Appl. Phsiol., 78: 1360-1368, 1995.

TARNOPOLSKY, L. J.; MacDOUGALL J. D.; ATKINSON S. A.; TARNOPOLSKY M. A.; SUTTON, J. R. Gender differences in substrate utilization for endurance exercise. J. Appl Physiol., 68: 302 - 8, 1990.

TREUTH, M.S.; ADOLPH, A.L. \& BUTTE, N.F.. Energy expenditure in children predicted from heart rate and activity calibrated against respiration calorimetry. Am. J. Physiol. Endocrinol. Metab.,275: E12 - 18, 1998.

YANNIS, P.; SMITH, I. Increased fat availability enhances the capacity os trained individuals to perform prolonged exercise. Med. Sci. Sports Exerc., Vol. 31, n 11 : 1570-1579, 1999.

WELKER, A.F. \& SOUZA, P.F.A.a. Efeito do exercício e do condicionamento físico na captação de glicose. Arq. Bras. Endocrinol. Metab, 46: supl.1, 505-505, 2002.

WELKER, A.F. \& SOUZA, P.F.A.b. Inibição da oxidação de ácidos graxos pela ingestão de açúcar simples após exercício físico. Arq. Bras. Endocrinol. Metab, 46: supl.1, 505-505, 2002.

WELKER, A.F. \& SOUZA, P.F.Ac. Efeito da ingestão de carboidrato sobre o metabolismo lipídico durante o repouso após exercício. Arq. Bras. Endocrinol. Metab, 46: supl.1, 505-505, 2002.

WELKER, A.F. Avaliação do perfil alimentar, nutricional e físico das atletas da equipe de Pólo Aquático da UnB. Brasília: UnB, 1999.67p. Monografia de Especialização. 